\title{
Lista preliminar de los Punctoideos de Rio Grande do Sul, Brasil, con descripción de dos especies nuevas (Mollusca, Gastropoda, Stylommatophora)
}

\author{
Sergio Eduardo Miquel ${ }^{1}$, Rina Ramírez ${ }^{2,3}$ \& José Willibaldo Thomé ${ }^{3}$ \\ ${ }^{1}$ División Invertebrados, Museo Argentino de Ciencias Naturales "Bernardino Rivadavia". Avenida Angel Gallardo 470,1405 \\ Buenos Aires, Argentina. E-mail: semsnail@muanbe.gov.ar; semsnail@yahoo.com.ar \\ ${ }^{2}$ Museo de Historia Natural, Universidad Nacional Mayor de San Marcos. Apartado 14-0434, Lima-14, Perú. \\ E-mail: rina_rm@yahoo.com \\ ${ }^{3}$ Laboratório de Malacologia, FaBio, Pontifícia Universidade Católica do Rio Grande do Sul. Avenida Ipiranga 6681, \\ Prédio12D, S340, 90619-900 Porto Alegre, Rio Grande do Sul, Brasil. E-mail: thomejw@pucrs.br
}

\begin{abstract}
Preliminar list of the Punctoidea from the State of Rio Grande do Sul, Brazil, with description of two new species (Mollusca, Gastropoda, Stylommatophora). The partial list of the land snails Punctoidea from the "Centro de Pesquisas e Conservação da Natureza Pró-Mata" (São Francisco de Paula, State of Rio Grande do Sul, Brazil) (CPCN-PM) is presented herein. This list includes seven species, two of them new. The CPCN-PM is located between 600 and $950 \mathrm{~m}$ of altitude; it has a temperate super humid to humid climate, with $2,250 \mathrm{~mm}$ of annual rainfall and means annual temperature of $14.5^{\circ} \mathrm{C}$; three main phytoecological regions are present -mixed ombrophile forest, dense ombrophile forest and savanna. The taxa found are: Rotadiscus amancaezensis (Hidalgo, 1869), Lilloiconcha tucumana (Hylton Scott, 1963), Radiodiscus cuprinus Fonseca \& Thomé, 2000, Radiodiscus tenellus Hylton Scott, 1957, Radiodiscus promatensis sp. nov., Zilchogyra gordurasensis (Thiele, 1927) and Zilchogyra zulmae sp. nov. The shell of $R$. promatensis sp. nov. is very small, almost flat, with very wide umbilicus, reniform aperture, teleoconch with spiral striae and 58-62 axial ribs on the body whorl. The shell of Z. zulmae sp. nov. is trochiform elevated, with five whorls with strong and widely spaced axial ribs (25-38), wide and perspective umbilicus and sub circular angled aperture. The charopid gastropod fauna from the CPCN-PM has three species known only from there ( $R$. cuprinus, $R$. promatensis sp. nov. and Z. zulmae sp. nov.), another one with previous known distribution only in Paraguay ( $R$. tenellus) and three common species (L. tucumana, $R$. amancaezensis and Z. gordurasensis) with distribution in other South American areas -mainly Yungas forests.

KEY WORDS. Charopidae, Helicodiscinae, Pulmonata, Rotadiscinae.
\end{abstract}

RESUMEN. Se presenta una lista parcial de gasterópodos terrestres Punctoideos del "Centro de Pesquisas e Conservação da Natureza Pró-Mata” (São Francisco de Paula, Estado de Rio Grande do Sul, Brazil) (CPCN-PM). Esta lista incluye siete especies, dos de ellas son nuevas. El CPCN-PM se localiza entre los 600 y 950 m de altitud; presenta clima templado superhúmedo a húmedo, lluvias de $2.250 \mathrm{~mm}$ anuales y temperatura promedio anual de $14,5^{\circ} \mathrm{C}$; están presentes tres regiones fitoecológicas principales, floresta ombrófila mixta, floresta ombrófila densa y sabana. Los taxones hallados son: Rotadiscus amancaezensis (Hidalgo, 1869), Lilloiconcha tucumana (Hylton Scott, 1963), Radiodiscus cuprinus Fonseca \& Thomé, 2000, Radiodiscus tenellus Hylton Scott, 1957, Radiodiscus promatensis sp. nov., Zilchogyra gordurasensis (Thiele, 1927) y Zilchogyra zulmae sp. nov. La conchilla de R. promatensis sp. nov. es muy pequeña, casi plana, con ombligo muy desarrollado, abertura reniforme, con estrías espirales en la teleoconcha y 58-62 costillas axiales en el último anfracto. La conchilla de Z. zulmae sp. nov. es troquiforme alta, con cinco anfractos con fuertes y espaciadas costillas axiales (25-38), ombligo amplio y perspectivo y abertura subcircular angulosa. La fauna de gasterópodos carópidos del CPCN-PM tiene tres especies conocidas sólo del área $(R$. cuprinus, $R$. promatensis sp. nov. y Z. zulmae sp. nov.), una, que se distribuye también en Paraguay ( $R$. tenellus) y tres (L. tucumana, R. amancaezensis y Z. gordurasensis), comunes a otras áreas de América del Sur, principalmente la región de las Yungas.

PALABRAS CLAVE. Charopidae, Helicodiscinae, Pulmonata, Rotadiscinae. 
El “Centro de Pesquisas e Conservação da Natureza Pró-Mata (CPCN-PM)" es un área natural protegida de propiedad de la "Pontifícia Universidade Católica do Rio Grande do Sul (PUCRS)" (Porto Alegre, Brasil), cuyo manejo y administración tiene por objetivo la investigación y conservación de ecosistemas característicos del sur de Brasil (Bertoletti \& Teixeira 1995). La prospección y análisis de su flora y su fauna comenzó en 1995 y continúa hasta el presente. Así, una importante colección de gasterópodos terrestres ya se encuentra depositada en el "Museu de Ciências e Tecnologia (MCP)", institución dependiente de PUCRS, también con sede en Porto Alegre.

En esta contribución se da a conocer la primera parte de una lista de gasterópodos pulmonados punctoideos de la familia Charopidae encontrados en el CPCN-PM. Esta lista incluyecinco especies conocidas y otras dos, correspondientes a los géneros Radiodiscus y Zilchogyra, nuevas para la ciencia. Aquí se sigue el sistema clasificatorio de Solem (1976), quien proponela división de los Punctoidea en tres familias: Punctidae, Endodontidae y Charopidae. Muchos de los Charopidae de Brasil fueron descriptos por THIELE (1927), cuyas descripciones someras e ilustraciones esquemáticas no facilitan las determinaciones precisas. Sin embargo, BAKER (1928) publicó su opinión acerca de la posible identidad de dichas especies en base a esas mismas descripciones e ilustraciones. Posteriormente, al gunas nuevas entidades fueron creadas por FonseCA \& Thоме́ (1994, 1995a, 2000) para el sur brasileño.

\section{MATERIALESY MÉTODOS}

Los ejemplaresfueron obtenidos principalmente en hojarasca y mantillo colectados en diversos puntos del CPCN-PM (Fig. 1) entre 1996 y 2002. Eventualmente, fueron obtenidos también por observación directa. Los 12 puntos de colecta se encuentran en las florestas ombrófila mixta y ombrófila densa. La mayoría de las muestras está constituída sólo por conchillas. Las conchillas se midieron $(\mathrm{mm})$, contándose el número de anfractos (№A) (EMBERTon 1985), que setransformó a sistema decimal para anál isis estadístico. Las medidas son: 1) diámetro mayor (DM), 2) altura (ALT), 3) diámetro del ombligo $(\mathrm{OMB}), 4)$ diámetro de la abertura (DA) y 5) al tura de la abertura (AA). Teniendo en cuenta las características conquiliológicas (número de anfractos, tamaño, forma de la abertura y del labro) todos los ejemplares se consideran adultos, excepto dos, correspondientes a Radiodiscus cuprinus y Zilchogyra zulmaesp. nov. Fueron realizados análisis multivariados (Análisis de Componentes Principales - ACP - y Funciones Discriminantes Canónicas - FDC) con los datos logaritmizados correspondientes a 40 especímenes adultos, mediante el programa SPSS 11.5. Los datos y figuras fueron procesados en Microsoft Excel.

Las fotografías de los ejemplares se obtuvieron a microscopio electrónico de barrido ambiental (modelo Philips XL 30 ESEM Series).

El material estudiado fue depositado en las siguientes instituciones: The Academy of Natural Sciences of Philadel phia
(ANSP) y Field Museum of Natural History (FMNH, Chicago) (Estados Unidos de América), Museo Argentino de Ciencias Naturales "Bernardino Rivadavia" (MACN-In) (Buenos Aires, Argentina), "Museu de Ciências e Tecnologia da PUCRS (MCP)" (Porto Alegre, Brasil), Museo de Historia Natural de la Universidad Nacional Mayor de San Marcos (MUSM) (Lima, Perú) y Senckenberg-Museum (SMF) (Frankfurt am Main, Alemania).

Ejemplares de especies previamente conocidas fueron obtenidos de las siguientes instituciones: MACN y Facultad de Ciencias Naturales y Museo de la Universidad Nacional de La Plata (MLP) (Argentina). Abreviatura utilizada: J. Thomé/ELM P (José Willibal do Thomé/Equipe do Laboratório de Malacologia da PUCRS).

\section{Caracterización del área (Fig. I)}

El “Centro de Pesquisas e Conservação da Natureza PróMata" tiene una superficie estimada de 4.500 hectáreas y está localizado entre los $29^{\circ} 27^{\prime} / 29^{\circ} 35^{\prime} \mathrm{S}, 50^{\circ} 08^{\prime} / 50^{\circ} 15^{\prime} \mathrm{W}$, en el municipio de São Francisco de Paula (estado de Rio Grande do Sul, Brasil). Desde el punto de vista geológico, está localizado en la Formación Serra Geral de la Provincia Paraná. Geomorfológicamente, el área está incluída en el borde este de la Región Planalto de Araucarias; se encuentra entre las cotas altimétricas de 600 y $950 \mathrm{~m}$, con declive moderadamentefuerte a muy fuerte. Su clima es templado superhúmedo a húmedo, condicionado por la latitud, altitud y las masas de aire marino. Estas masas de aire penetran desde el océano, cargadas de humedad, provocando lluvias bien distribuídas duranteel año, además de propiciar una casi permanente nubosidad junto al borde del planalto. El total pluviométrico anual se sitúa en torno de los $2.250 \mathrm{~mm}$, la humedad media es de $84 \%$ y las temperaturas medias máxima y mínima son de $25,0^{\circ} \mathrm{C}$ y $5,7^{\circ} \mathrm{C}$, respectivamente, con un promedio anual de $14,5^{\circ} \mathrm{C}$ (BERTOLETTI \& TEIXEIRA 1995).

En el Centro aparecen tres regiones fitoecológicas: floresta ombrófila mixta, floresta ombrófila densa y sabana, con la presencia de algunas especies de la región de la floresta estacional semidecidual. La primera es el área mejor conservada del Centro, su composición consta de 350 especies, 13\% de las cuales son exclusivas; una de las más características es Araucaria angustifolia (Bertol.) Kuntze. La segunda región (también entre 600 y $900 \mathrm{~m}$ de altitud) presenta una floresta exuberante de cubierta cerrada y densa. Esta floresta fue Ilamada, originalmente, Mata Atlántica, por desarrollarse a lo largo del Océano Atlántico. Su composición florística consta de 700 especies, siendo $50 \%$ de ellas exclusivas. La tercera región se extiende en terrenos de relieve suavemente ondulado en el noroeste del Centro, su vegetación es gramíneo-leñosa, con numerosas compuestas y leguminosas. En esta área aparece, diferenciada, una comunidad vegetal Ilamada "mata nebular", discontínua y a modo de una faja estrecha, que consta de vegetación arbórea baja, raquítica, con copas densas y desarrollo de musgos y líquenes (Bertoletti \& TeiXeIRA 1995).

Revista Brasileira de Zoologia 21(4): 925-935, dezembro 2004 


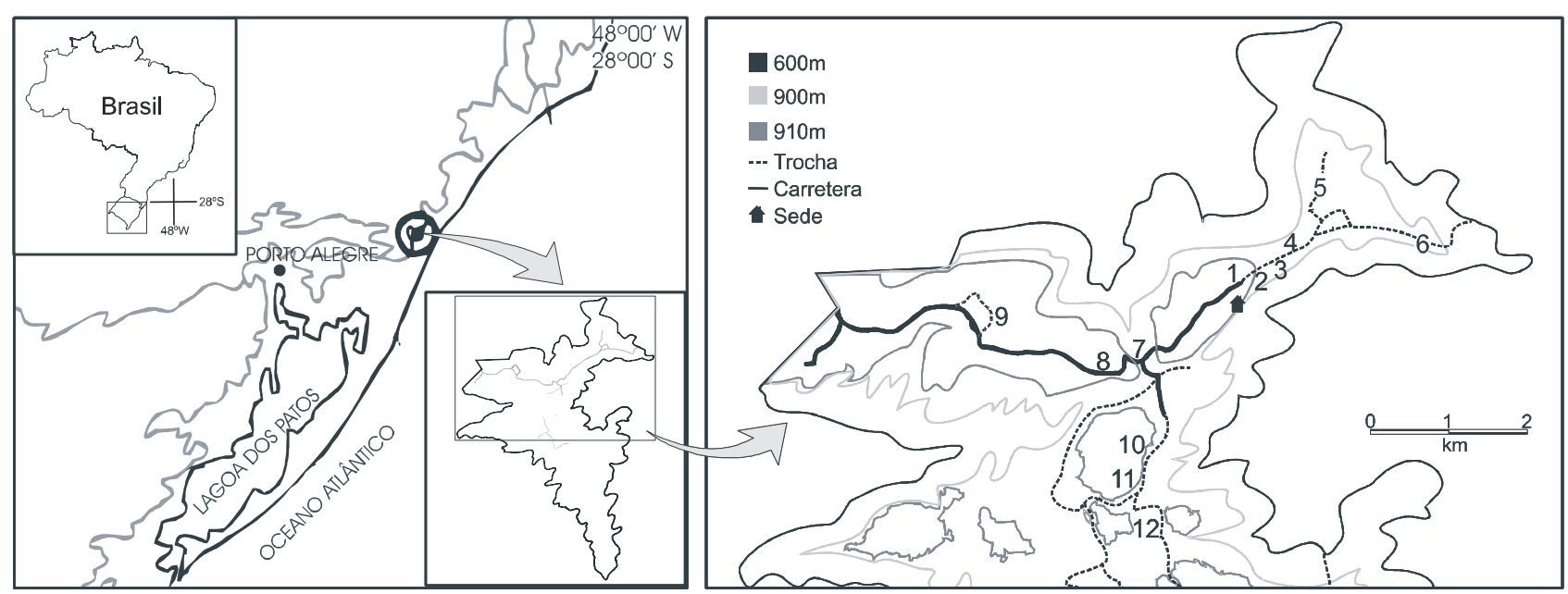

Figura 1. Ubicación del "Centro de Pesquisas e Conservação da Natureza Pró-Mata” y áreas de muestreo: (1) Trilha das Cobras; (2) Mollusca II, Punto 14; (3) Área 1; (4) Mollusca I; (5) Área 4/Nossa Mata/Mata Atlântica; (6) Taquaral; (7) Área 5: Serraria A/Mollusca II, Punto 9; (8) Área 2; (9) Área 3; (10) Área 8: Serraria B; (11) Mollusca II, Punto 19; (12) Serraria C.

Una parte del área ocupada por el Centro ha sufrido, desde tiempos históricos, una importante explotación de sus recursos naturales, principalmente de ejemplares de Araucaria angustifolia, completada con desmonte y quema de restos vegetales, reforestación con Pinus sp., y establecimiento de labores pecuarias y agrícolas. Una vez que estas actividades cesaron, con la compra del área en 1993, comenzó a desarrollarse en las zonas más al teradas una vegetación secundaria, actualmente en diferentes estadíos de sucesión (Bertoletti \& TeIXeIrA 1995).

De acuerdo al esquema fitogeográfico de CABRERA (1958), esta región se halla comprendida en Ia Provincia Subtropical Oriental, distrito que se extiende desde el este de Paraguay, noroeste de Argentina y extremo sur de Brasil, prolongándose en forma de galerías a lo largo de los ríos Paraná y Uruguay hasta el Río de la Plata.

\section{RESULTADOS}

Hasta el momento las especies encontradas en el CPCNPM son las siguientes:

\section{Charopidae Hutton, 1884 Rotadiscinae Baker, 1927 Rotadisaus Pilsbry, 1926}

Especie tipo: Helix hermanni Pfeiffer, 1866.

\section{Rotadisas amancaezensis (Hidalgo, 1869), comb. nov.}

Helix amancaezensis Hidalgo, 1869: 411; Hidalgo, 1870: 38, pl. 6, fig. 3.

Stephanoda jujuyensis Hylton Scott, 1948: 251, fig. 4.

Ptychodon (Ptychodon) amancaezensis; Fernández, 1973: 43; Fonseca \& Thomé, 1993b: 72.
Ptychodon (Ptychodon) jujuyensis; Fonseca \& Thomé, 1993b: 73. Localidad típica de H. amancaezensis: Perú, Amancaez (sic) $=$ Amancaes.

Localidad típica de S. jujuyensis: Argentina, Ciudad de Jujuy, barranca del río Chico, a la sombra de espesos matorrales. Distribución. Argentina (Catamarca, Jujuy, Misiones, Salta, Tucumán); Brasil (sur), Perú (Lima, Cuzco).

Comentarios. En relación a la lista sinonímica, aquí se sigue parcialmente el criterio de CLIMo (1989). WEYRAuCH (1965b) señala las sinonimias de esta especie con Endodonta discoidea Thiele, 1927 y S. jujuyensis, combinándola con Ptychodon Ancey, 1888. Climo (1989) las reitera y las relaciona a Rotadiscus. Este autor agrega Helix hermanni Pfeiffer, 1866, lo que no se ha podido confirmar. Sin embargo, E. discoidea no parece corresponderse al resto de las especies sinonimizadas.

La localidad tipo de R. amancaezensis está a una distancia de $4 \mathrm{~km}$ del centro de la ciudad de Lima (Perú). Luego de su descripción, no fue citado un nuevo dato de esta especie hasta 1965, cuando WeYrauch (1965a) publica dos nuevas localidades: Infiernillo (Lima, 3360 m) y Kachuna (Huancaro, Cuzco, $3500 \mathrm{~m}$ ), ambas en Perú, asegurando que no se lo encuentra en las lomas, ni tampoco por debajo de los $2850 \mathrm{~m}$ de altitud. Uno de los autores (RR) la encontró en el ecosistema de lomas, El Lúcumo (1215'18"S, 7647'19,8"W ), local idad ubicada a 35 $\mathrm{km}$ al SE de Amancaes.

Material es estudiados. 2 ejs (1, dañado), BrASIL, Rio Grande do Sul: São Francisco de Paula, CPCN-PM , 29²9'04,4"S, 50¹2'58,7"W, J. Thomé/ELMP leg., 23.III.1997, М CP 6991; 1 ej., CPCN-PM 29²8'67,2"S, 50¹0'11,9"W. Mollusca I, J. Thomé/ELMP leg., 19.V.1996, MACN-In 35.854 (ex. MCP 6889a); 1 ej. CPCN-PM, J. Thomé/ELMP leg., MUSM 4015 (ex. MCP 6900b); 1 ej., CPCN-PM, J. Thomé/ELMP leg., FMNH 
306.260 (ex. MCP 6905a). 1 ej., Argentina, Jujuy: Río Chico, 22.II.1945, Holotipo, MLP s/n; 1 ej. Argentina, Jujuy, Río Chico, 22.II.1945, Paratipo, MLP s/n.

Las principales dimensiones de ejemplares de esta especie aparecen en la tabla I.

Tabla I. Dimensiones de Rotadiscus amancaezensis (en mm).

\begin{tabular}{lcccccc}
\hline & DM & ALT & OMB & DA & AA & $N^{\circ}$ A \\
\hline FMNH 306.260 & 1,64 & 0,69 & 0,63 & 0,24 & 0,75 & $43 / 4$ \\
MACN-In 35.854 & 1,21 & 0,62 & 0,44 & 0,25 & 0,62 & $33 / 4$ \\
MCP 6991 & 1,09 & 0,59 & 0,31 & 0,16 & 0,56 & $31 / 2$ \\
MUSM 4015 & 1,02 & 0,53 & 0,31 & 0,22 & 0,53 & $33 / 4$ \\
\hline
\end{tabular}

\section{Helicodiscinae Baker, 1927}

Lilloicondha Weyrauch, 1965

Especietipo: Austrodiscus superbus tucumanus Hylton Scott, 1963

\section{Lilloicondha tuamana (Hylton Scott, 1963)}

Austrodiscus superbus tucumanus Hylton Scott, 1963: 53, fig. 3. Lilloiconcha tucumana; Weyrauch, 1965: 127, Abb. 1; Fonseca \& Thomé, 1993a: 103.

Localidad típica. Argentina, Tucumán, Horco Molle. Distribución. Argentina (Misiones, Tucumán) y Brasil (sur).

Comentario. Según Weyrauch (1965a, b) las especies del género están presentes en bosques pluviales húmedos subtropicales del nortedeArgentinay, tal vez, en el sur de Bolivia y Brasil, a una altitud de 600 a $1400 \mathrm{~m}$, en el suelo del bosque (en hojarasca). Aquí se confirma su presencia en el sur brasileño.

Materiales estudiados. 1 ej., BRASIL, Rio Grande do Sul: São Francisco de Paula, CPCN-PM, 29²8'60,6"S, 50¹0'04,9"W, J. Thomé/ELMP leg., 30.III.1996, MCP 6954; 1 ej. (dañado), CPCNPM, Mata Atlântica, J. Thomé/ELMP leg., 7.IV.2001, MCP 7940; 1 ej. (dañado), CPCN-PM, Taquaral, J. Thomé/ELMPleg., 7.IX.2002, MACN-In 35.855 (ex. MCP 8028); 1 ej., CPCN-PM, Nossa Mata, J. Thomé/ELMP leg., 13.X.2002, MACN-In 35.856 (ex. MCP 8029); 1 ej., CPCN-PM , área 4, Nossa Mata, J. Thomé/ELMPleg., 13.X.2001, MUSM 4016 (ex. MCP 8030); 1 ej., CPCN-PM, Nossa Mata, J. Thomé/ELMP leg., 13.X.2002, SMF 325.466 (ex. MCP 8031).

Las principales dimensiones de ejemplares de esta especie aparecen en la tabla II.

Tabla II. Dimensiones de Lilloiconcha tucumana (en mm).

\begin{tabular}{lcccccc}
\hline & DM & ALT & OMB & DA & AA & $N^{\circ} A$ \\
\hline MACN-In 35.855 & 2,65 & 2,09 & 0,61 & 1,02 & 1,02 & dañado \\
MACN-In 35.856 & 2,44 & 1,68 & 0,56 & 0,92 & 0,97 & $53 / 4$ \\
MCP 7940 & 2,29 & 1,58 & 0,66 & 0,61 & 0,81 & $51 / 4$ \\
MUSM 4016 & 2,85 & 2,44 & 0,76 & 1,02 & 0,85 & $61 / 2$ \\
SMF 325.466 & 2,29 & 1,63 & 0,81 & 0,81 & 0,76 & $53 / 4$ \\
\hline
\end{tabular}

\section{Radiodisaus Pilsbry \& Ferriss, 1906}

Especie tipo: Radiodiscus millecostatus Pilsbry \& Ferriss, 1906.

Radiodisaus aprinus Fonseca \& Thomé, 2000

Radiodiscus cuprinus Fonseca \& Thomé, 2000: 12, figs 1-6.

Localidad típica. Brasil, Rio Grande do Sul, Cristal, Parque Bento Gonçalves (en las márgenes de la ruta BR 116).

Distribución. Brasil (sur).

Materiales estudiados. 1 ej., BRASIL, Rio Grande do Sul: São Francisco de Paula, CPCN-PM, 29²8'21,6"S, 5009'42,5”W, 750 m, J. Thomé/ELMP leg., 28.VI.1997, MCP 6785; 1 ej., CPCN-PM, Taquaral, J. Thomé/ELMPleg., 7.IX.2002, MCP 8016; 1 ej., CPCNPM (área 4), J.Thomé/ELMP leg., 13.X.2002, MCP 8017; 2 ejs, CPCN-PM, Trilha das cobras, J. Thomé/ELMP leg., 25.VIII.2002, MACN-In 35.857 (ex. MCP 8032); 2 ejs, CPCN-PM, área 3, J. Thomé/ELMP leg. , 8.XII.2001, M ACN-In 35.858 (ex. MCP 8033); 1 ej., CPCN-PM, Taquaral, J.Thomé/ELMP leg., 7.IX.2002, ANSP 411.191 (ex. MCP 8034); 1 ej., CPCN-PM, 29²8'28,9"S, 5009'38,0"W, 914 m, J. Thomé/ELMP leg., 1.VI.1997, FMNH 306.261 (ex. MCP 6709).

Las principales dimensiones de ejemplares de esta especie aparecen en la tabla III.

\section{Radiodisas tendlus Hylton Scott, 1957}

Figs 2-4

Radiodiscus tenellus Hylton Scott, 1957: 83; Quintana, 1982:

77; Fonseca \& Thomé, 1993a: 102.

Localidad típica. Paraguay, Villarrica.

Distribución. Paraguay, Brasil (sur).

M aterial es estudiados. 1 ej., BRASIL, Rio Grande do Sul: São Francisco dePaula, CPCN-PM, 29²8'21,6"S, 5009'42,5"W, 750 $\mathrm{m}$, Mollusca II, punto 9, J. Thomé/ELMP leg., 28.VI.1997, MCP 6787b; 3 ejs (uno, dañado), CPCN-PM, Taquaral, J. Thomél ELMP leg., 7.IX.2002, MCP 8018; 1 ej. (dañado), CPCN-PM, área 1, J. Thomé/ELMP leg., 25.VIII.2002, MCP 8019; 3 ejs (dañados), CPCN-PM (Serraria C), J. Thomé/ELMP leg., 8.IX.2002, MCP 8020; 2 ejs, CPCN-PM, Trilha das cobras, J. Thomé/ELMP leg., 25.VIII.2002, MACN-In 35.859 (ex. MCP 8035); 2 ejs (1, dañado), CPCN-PM, Taquaral, J. Thomé/ELMP leg., 7.IX.2002, MACN-In 35.860 (ex. MCP 8036); 2 ejs, CPCNPM, área 3, J. Thomé/ELMP leg., MACN-In 35.861 (ex. MCP 8037); 1 ej., CPCN-PM, 2328'27,5"S, 5009'39,6"W, 983 m, J. Thomé/ELMP leg., 17.IV.1998, MUSM 4017 (ex. MCP 6820); 1 ej., CPCN-PM, 29²8'49,7"S, 50¹0'32,0"W, 941 m, J. Thomél ELMP leg., 12.IX.1998, ANSP 411.192 (ex. MCP 6847); 1 ej., CPCN-PM, 2328'28,9"S, 5009'38,0"W, 914 m, J. Thomé/ELMP leg., 1.VI.1997, FMNH 306.262 (ex. MCP 8041); 1 ej., CPCNPM, 29²8'27,5"S, 5009'39,7"W, 915 m, J. Thomé/ELMP leg., 31.V.1997, SMF 325.467 (ex. MCP 6700); 1 ej.; Paraguay, Villarrica; F. Schade, 1931 leg., Holotipo; MACN-In 19.991-1.

Las principales dimensiones de ejemplares de esta especie aparecen en la tabla IV. 

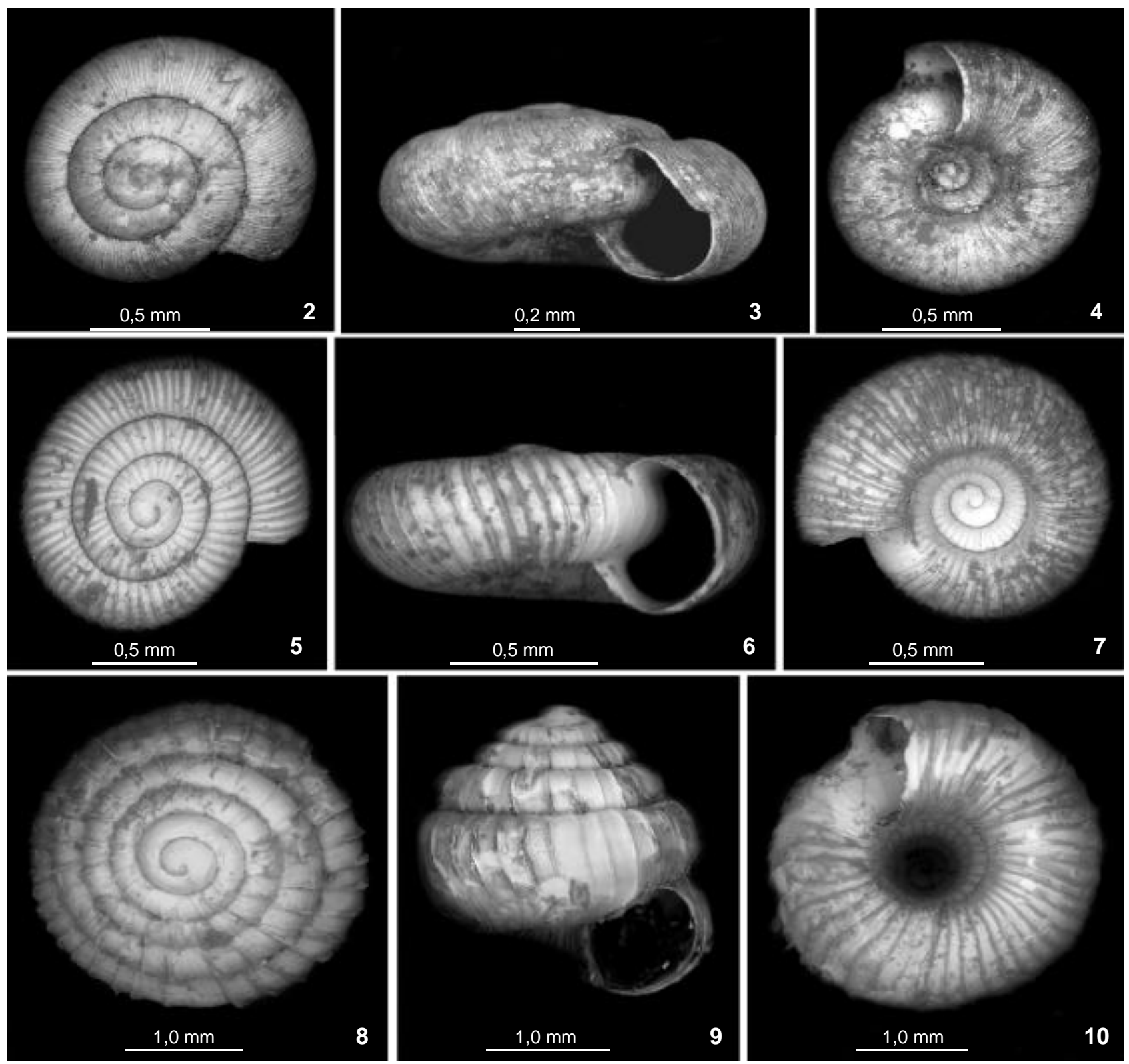

Figuras 2-10. (2-4) Radiodiscus tenellus, holotipo (MACN-In 19991-19), vistas: (2) apical, (3) apertural, (4) umbilical; (5-7) Radiodiscus promatensis sp. nov., holotipo (MCP 8038), vistas: (5) apical, (6) apertural, (7) umbilical; (8-10) Zilchogyra zulmae sp. nov., holotipo (MCP 8042), vistas: (8) apical, (9) apertural, (10) umbilical.

\section{Radiodisaus promatensis sp. nov.}

\section{Figs 5-7}

Diagnosis. Radiodiscus muy pequeño, casi plano, con ombligo muy desarrollado, abertura reniforme, con estrías espirales en la teleoconcha y 58-62 costillas axiales en el último anfracto.

Descripción. Radiodiscus de muy pequeño tamaño (en- tre 1,20 y 1,60 mm de diámetro mayor), discoide, casi plano, umbilicado, con algo más de 4 anfractos totales, protoconcha de $13 / 4$ anfractos, ápice que apenas sobresale de perfil, sutura profunda, abertura amplia y reniforme, ombligo amplio (40\% respecto del diámetro mayor) y perspectivo. Último anfracto con 58-62 costillas axiales, apareciendo 22 por $\mathrm{mm}$ en la última parte, bien marcadas, espacios intercostulares recorridos por cordones filiformes axiales, incisuras transversas surcan toda 
Tabla III. Dimensiones de Radiodiscus cuprinus (en mm).

\begin{tabular}{lcccccc}
\hline & DM & ALT & OMB & DA & AA & N$^{\circ}$ A \\
\hline ANSP 411.191 & 1,19 & 0,65 & 0,22 & 0,26 & 0,42 & $41 / 8$ \\
FMNH 306.261 & 0,95 & 0,49 & 0,19 & 0,28 & 0,40 & $23 / 4$ \\
MACN-In 35.857 & 1,19 & 0,67 & 0,20 & 0,34 & 0,51 & 4 \\
MACN-In 35.858 & 1,33 & 0,71 & 0,22 & 0,30 & 0,49 & $41 / 4$ \\
MCP 8016 & 1,50 & 0,91 & 0,20 & 0,42 & 0,59 & $41 / 8$ \\
MCP 8017 & 1,13 & 0,63 & 0,20 & 0,30 & 0,49 & 4 \\
\hline
\end{tabular}

la superficie, color castaño-amarillento. La protoconcha consta de numerosos cordones filiformes espirales, que hacia el final son atravesadas por incisuras transversas, formando un delicado retículo.

Comparaciones. Sus caracteres diagnósticos la diferencian claramente de las restantes especies de Radiodiscus, en particular por su morfología aplanada y su pequeño tamaño. De R. millecostatus, especie tipo, se diferencia porque ésta presenta mayor cantidad de costillas axiales y abertura subcircular descendente; R. bequaerti Flórez Bustamante, 1968; R. costellifer Hylton Scott, 1957; R. ditzleri Baker, 1925; R. florezi Weyrauch, 1965; R. lateumbilicatus Weyrauch, 1966 y R. peruvianus Crawford, 1939 se diferencian de la nueva especie por presentar menor número de costillas axiales, espira al go desarrollada y abertura circular o subcircular; R. coarctatus Hylton Scott, 1979; R. compactus (Suter, 1900); R. coppingeri (Smith, 1881); R. cuprinus Fonseca \& Thomé, 2000; R. flammulatus Hylton Scott, 1975; R. katiae Hylton Scott, 1948; R. magellanicus (Smith, 1881); R. proameri Baker, 1930; R. quillajicola Vargas-Almonacid, 2000; R. tenellus Hylton Scott, 1957; R. thomei Weyrauch, 1965; R. titicacensis Hylton Scott, 1957; R. vazi Fonseca \& Thomé, 1994 y R. wigodzinskyi Weyrauch, 1965 presentan un número mayor de costillas axiales, rasgo que se combina con: espira más desarrollada, ombligo más pequeño y/ o abertura circular o subcircular; R. andium Pilsbry, 1944; R. australis Hylton Scott, 1970; R. crenulatus Hylton Scott, 1963; R. golbachi Weyrauch, 1965; R. mariae Pilsbry, 1921 y R. microhelix Haas, 1951 se diferencian por la combinación de los siguientes caracteres: mayor desarrollo de la espira, ombligo más pequeño y/o abertura circular o subcircular.

Localidad típica. Brasil, Rio Grande do Sul: São Francisco de Paula, en el Centro de Pesquisas e Conservação da Natureza PRO-MATA (CPCN-PM), de la Pontifícia Universidade Católica do Rio Grande do Sul (PUCRS), $29^{\circ} 27^{\prime} / 29^{\circ} 35^{\prime} \mathrm{S}, 50^{\circ} 08^{\prime} /$ $50^{\circ} 15^{\prime} \mathrm{W}$, entre 600 y $950 \mathrm{~m}$.

Derivación del nombre: de PRÓ-MATA, de donde procede el material.

Distribución: Brasil (sur).

Materiales estudiados. Holotipo: BrasIL, Rio Grande do Sul: São Francisco de Paula, CPCN-PM, área 5, Serraria A, J. Thomél ELMP leg., 14.X.2001, MCP 8038. Paratipos. 2 ejs, CPCN-PM, $30^{\circ} 03^{\prime} 09,2^{\prime \prime} \mathrm{S}, 51^{\circ} 10^{\prime} 33,6^{\prime \prime} \mathrm{W}$, punto 9, J. Thomé/ELMP leg.,
Tabla IV. Dimensiones de Radiodiscus tenellus (en mm).

\begin{tabular}{lcccccc}
\hline & DM & ALT & OMB & DA & AA & $N^{\circ}$ A \\
\hline ANSP 411192 & 1,01 & 0,49 & 0,24 & 0,30 & 0,40 & $31 / 2$ \\
FMNH 306.262 & 0,93 & 0,49 & 0,24 & 0,24 & 0,40 & $31 / 4$ \\
MACN-In 35.859 & 1,19 & 0,55 & 0,32 & 0,26 & 0,45 & 4 \\
MACN-In 35.860 & 1,09 & 0,59 & 0,22 & 0,30 & 0,49 & $31 / 8$ \\
MCP 6787b & 1,29 & 0,71 & 0,44 & 0,30 & 0,45 & $41 / 4$ \\
MCP 8018 & 0,91 & 0,45 & 0,22 & 0,20 & 0,36 & $31 / 8$ \\
MCP 8018 & 1,09 & 0,53 & 0,24 & 0,26 & 0,42 & $31 / 2$ \\
MUSM 4017 & 0,93 & 0,47 & 0,26 & 0,22 & 0,34 & $31 / 2$ \\
SMF 325.467 & 0,93 & 0,44 & 0,26 & 0,26 & 0,34 & $31 / 2$ \\
\hline
\end{tabular}

12.IX.1998, MCP 6839a; 2 ejs, CPCN-PM, área 5, Serraria A, J. Thomé/ELMPleg., 14.X.2001, MACN-In 35.863 (ex. MCP 8021a); 1 ej., CPCN-PM, área 5, Serraria A, J. Thomé/ELMP leg., 14.X.2001, MACN-In 35.864 (ex. MCP 8021b); 1 ej., CPCN-PM, $29^{\circ} 29^{\prime} 09,3^{\prime \prime} \mathrm{S}, 50^{\circ} 11^{\prime} 10,6^{\prime \prime} \mathrm{W}$, Mollusca II punto 19, J. Thomél ELMP leg., 21.XI.1998, ANSP 411.193 (ex. MCP 6852); 1 ej., CPCN-PM, 3003'09,2"S, 5110'33,6”W, Mollusca II punto 14, J. Thomé/ELMP leg., 21.IX.1998, FMNH 306.263 (ex. M CP 6839); 1 ej., CPCN-PM, área 4, J. Thomé/ELMP leg., 13.X.2001, SMF 325.468 (ex. MCP 8039). Otros: 2 ejs (dañados), CPCN-PM, $30^{\circ} 03^{\prime} 39,2^{\prime \prime} \mathrm{S}, 51^{\circ} 10^{\prime} 33,6^{\prime \prime} \mathrm{W}$, Mollusca II, punto 14, J. Thomél ELMP leg., 12.IX.1998, MCP 6841; 5 ejs (dañados), CPCN-PM, 29²9'39,7"S, 50¹1'10,6"W, 999 m, Mollusca II, punto 19, J. Thomé/ELM P leg., 21.XI.1998, MCP 6851; 1 ej. (dañado), CPCNPM , área 5, Serraria A, J. Thomé/ELM P leg., 14.X.2001, M CP 8022.

Las principal es dimensiones de ejemplares de esta especie aparecen en la tabla V.

\section{Zldhogyra Weyrauch, 1965}

Especie tipo: Helix costellata d'Orbigny, 1835

\section{Zlchogyra gordurasensis (Thiele, 1927)}

Endodonta gordurasensis Thiele,1927: 321, est. 26, fig. 19. Austrodiscus golbachi Hylton Scott, 1963: 51, fig. 2.

Zilchogyra gordurasensis; Weyrauch, 1965a: 122.

Zilchogyra golbachi; Weyrauch, 1965a: 122.

Zilchogyra gordurasensis; Meyer \& Weyrauch, 1966: 44, fig. 24, 6. Trochogyra (Trochogyra) golbachi; Fonseca \& Thomé, 1993a: 103.

Localidad típica de Zilchogyra gordurasensis. BRASIL, Minas Gerais: Gorduras (junto a Belo Horizonte).

Localidad típica de Austrodiscus golbachi. Argentina, Tucumán: Quebrada de Cainzo.

Distribución. Argentina, Paraguay, Brasil (sur).

Comentarios. Citada para Villarrica (Paraguay) por HyLton ScotT (1963), pero el ejemplar del lote MACN-In 19.991 correspondiente a esta localidad, citado por la autora, no ha sido hallado. QuinTANA (1982) incluyeaZ. golbachi en lasinonimia de Endodonta discoidea Thiele, 1927 y Stephanoda jujuyensis Hylton Scott, 
Tabla V. Dimensiones de Radiodiscus promatensis (en $\mathrm{mm}$ ).

\begin{tabular}{|c|c|c|c|c|c|c|}
\hline & DM & $\mathrm{ALT}$ & OMB & $\overline{\mathrm{DA}}$ & $\overline{A A}$ & $\mathrm{~N}^{\circ} \mathrm{A}$ \\
\hline Holotipo MCP 8038 & 1,48 & 0,67 & 0,55 & 0,34 & 0,65 & $33 / 4$ \\
\hline Paratipo MACN-In 35.864 & 1,39 & 0,61 & 0,49 & 0,30 & 0,57 & $33 / 4$ \\
\hline Paratipo ANSP 411193 & 1,48 & 0,69 & 0,53 & 0,28 & 0,40 & $31 / 2$ \\
\hline Paratipo FMNH 306.263 & 1,29 & 0,57 & 0,49 & 0,30 & 0,51 & 4 \\
\hline Paratipo MACN-In 35.863 & 1,56 & 0,67 & 0,45 & 0,32 & 0,63 & 4 \\
\hline Paratipo MCP 8021a & 1,43 & 0,42 & 0,44 & 0,22 & 0,40 & $31 / 4$ \\
\hline Paratipo SMF 325.468 & 1,44 & 0,67 & 0,53 & 0,40 & 0,59 & 4 \\
\hline
\end{tabular}

1948, pero ambas especies se consideran diferentes deZ. gordurasensis. En este estudio mantenemos la combinación establecida por Weyrauch (1965a) hasta tanto se revisen las identidades de Zilchogyra y Trochogyra Weyrauch, 1965a.

Materiales estudiados. 1 ej., BRASIL, Rio Grande do Sul: São Francisco de Paula, CPCN-PM, Serraria C, J. Thomé/ELM P leg., 8.IX.2002, MCP 8023; 11 ejs (3, dañados), CPCN-PM , J. Thomél ELMP leg., MCP 6906b; 5 ejs, CPCN-PM, J. Thomé/ELMP leg., MACN-In 35.862 (ex. MCP 6906a); 2 ejs, CPCN-PM, J. Thomél ELMP leg., MUSM 4018 (ex. MCP 6906c); 1 ej., CPCN-PM, 29²9'04,4"S, 50¹2'58,7"W, 891 m, J. Thomé/ELMP leg., 22.III.1997, SMF 325.469 (ex. MCP 7297); 1 ej., ArgentinA, Tucumán: Quebrada de Cainzo, M.I. Hylton Scott leg., 1963, MACN-In 26.634; 1 ej., R. Golbach leg., Holotipo, MLP s/n; 2 ejs, Jujuy: San Salvador, Banda de los Perales, M. Birabén leg., XII.1950, Paratipos, MLP s/n.

Las principal es dimensiones de ejemplares de esta especie aparecen en Ia tabla VI.

Tabla VI. Dimensiones de Zilchogyra gordurasensis (en mm).

\begin{tabular}{lcccccc}
\hline & DM & ALT & OMB & DA & AA & $N^{\circ}$ A \\
\hline MACN-In 35.862 & 2,22 & 1,19 & 0,75 & 0,50 & 0,72 & $41 / 4$ \\
MACN-In 35.862 & 2,09 & 1,03 & 0,68 & 0,50 & 0,72 & 4 \\
MACN-In 35.862 & 2,03 & 1,00 & 0,66 & 0,37 & 0,78 & 4 \\
MACN-In 35.862 & 1,87 & 1,00 & 0,56 & 0,47 & 0,75 & $33 / 4$ \\
MUSM 4018 & 1,68 & 0,85 & 0,57 & 0,42 & 0,71 & $33 / 4$ \\
MUSM 4018 & 1,84 & 0,95 & 0,63 & 0,65 & 0,79 & $33 / 4$ \\
SMF 325.469 & 1,68 & 0,83 & 0,45 & 0,30 & 0,67 & $33 / 4$ \\
\hline
\end{tabular}

\section{Zlchogyra zulmae sp. nov.}

Figs 8-10

Diagnosis. Conchilla troquiforme alta, con 5 anfractos con fuertes y escasas costillas axiales (25-38), ombligo amplio y perspectivo y abertura subcircular angulosa.

Descripción. Conchilla troquiforme alta, umbilicada, 5$53 / 4$ anfractos, ápice prominente, espira elevada; $13 / 4$ vueltas nepiónicas lisas; la teleoconcha está ornamentada de fuertes costillas espaciadas, contándose 25-38 en el último anfracto, con 5-6 costillas axiales por $\mathrm{mm}$ en el última parte del último anfracto. En los espacios intercostillares aparecen cordones filiformes apretados y tenues, en tanto que delicadas incisuras surcan espiralmente la conchilla. Entre las costillas mayores pueden aparecer otras de aspecto irregular que no llegan a tener el desarrollo de las principales, pero son de igual naturaleza. Anfractos convexos con sutura bien marcada. Abertura subcircular, inclinada, angulosa en su base y al go descendente. Ombligo amplio, de 1/3 en relación al diámetro mayor, profundo y perspectivo.

Comparaciones. Zilchogyra costellata presenta una conchilla cónica baja, de 4,5 mm y 41/2 anfractos, con 52 a 60 costillas axiales en el último anfracto; $Z$. michaelseni (Strebel, 1907) es cónica baja, con 84 costillas axialesen el último anfracto, sin estriación espiral, con perióstraco con bandas pardas y claras; Z. hyltonscottae Weyrauch, 1965 es cónico-deprimida, de 2,3 $\mathrm{mm}$, con 14 costillas axiales en $1 \mathrm{~mm}$ en el último anfracto, sin estriación espiral, y último anfracto dilatado en su porción final; Z. calchaqui Hylton Scott, 1975 es heliciforme, presenta 31/2 anfractos, abertura circular, ombligo de $1 / 5$ en relación al diámetro mayor, con 14 a 18 costillas axiales en $1 \mathrm{~mm}$ en el último anfracto; $Z$. descendens Weyrauch in Flórez Bustamante, 1968 es discoidal, de espira muy baja, con 41/2 anfractos y 37 costillas axiales en el último anfracto. El resto de las especies de Zilchogyra son planas o casi planas (Z. gordurasensis; Z. paulistana Hylton Scott, 1973; Z. materiae Hylton Scott 1972; Z. solemi Valdovinos \& Stuardo, 1989 y Z. franzi Weyrauch, 1965).

Localidad típica. BRASIL, Rio Grande do Sul: São Francisco de Paula, en el Centro de Pesquisas e Conservação da Natureza PRO-MATA, (CPCN-PM), de la Pontifícia Universidade Católi-

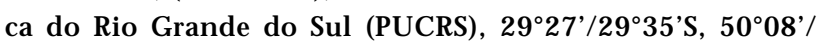
$50^{\circ} 15^{\prime} \mathrm{W}$, entre 600 y $950 \mathrm{~m}$.

Derivación del nombre. Dedicada a la Dra. Zulma Ageitos de Castellanos, destacada malacóloga argentina y primera directora del Convenio Binacional Brasil/Argentina por la contraparte argentina.

Distribución. Brasil: (sur).

Materiales estudiados. Holotipo: 1 ej., BrasIL, Rio Grande do Sul: São Francisco de Paula, CPCN-PM, área 8, Serraria B, J. Thomé/ELMP leg., 24.VIII.2002; M CP 8042. Paratipos: 1 ej., BrASIL, Rio Grande do Sul: São Francisco de Paula, CPCN-PM, área 5, J. Thomé/ELMP leg., 9.XII.2001, MCP 8024; 1 ej., CPCN-PM, área 5, serraria A, J. Thomé/ELM P leg., 24.VIII.2002, MCP 8025; 2 ejs, CPCN-PM, área 5, J. Thomé/ELMP leg., 9.XII.2001, MACNIn 35.866 (ex. MCP 8040); 1 ej., CPCN-PM, 3003'39,2"S, $51^{\circ} 10^{\prime} 33,6^{\prime \prime} \mathrm{W}$, Mollusca II, punto 14, J. Thomé/ELMP leg., 12.IX.1998, MACN-In 35.867 (ex. MCP 8026); 1 ej., CPCN-PM, $30^{\circ} 03^{\prime} 39,2^{\prime \prime} \mathrm{S}, 51^{\circ} 10^{\prime} 33,6^{\prime \prime} \mathrm{W}$, Mollusca II, punto 14, J. Thomé/ ELMP leg., 12.IX.1998, ANSP 411.194 (ex. MCP 6799). Otro: 1 ej. (dañado), CPCN-PM, área 5, serraria A, J. Thomé/ELMP leg., 7.XII.2001, MCP 6840b.

Las principales dimensiones de ejemplares de esta especie aparecen en la tabla VII. 
Tabla VII. Dimensiones de Zilchogyra zulmae (en mm).

\begin{tabular}{|c|c|c|c|c|c|c|}
\hline & $\mathrm{DM}$ & ALT & $\mathrm{OMB}$ & DA & AA & $\mathrm{N}^{\circ} \mathrm{A}$ \\
\hline Holotipo MCP 8042 & 2,88 & 2,28 & 0,84 & 0,72 & 1,06 & $43 / 4$ \\
\hline Paratipo MACN-In 35.866 & 3,26 & 2,80 & 1,27 & 1,02 & 1,27 & $51 / 2$ \\
\hline Paratipo MACN-In 35.867 & 1,56 & 0,97 & 0,47 & 0,37 & 0,72 & $31 / 4$ \\
\hline Paratipo ANSP 411194 & 3,10 & 2,54 & 1,02 & 0,81 & 1,07 & $53 / 4$ \\
\hline Paratipo MCP 8024 & 2,85 & 2,28 & 1,02 & 0,76 & 1,07 & 5 \\
\hline
\end{tabular}

\section{A nálisis conquiliomorfométrico}

El análisis morfométrico de las conchillas de ejemplares adultos de los siete taxones estudiados permitió diferenciar inequívocamente a las dos nuevas especies (R. promatensis y $Z$. zulmae) de las otras de sus respectivos géneros. Tanto el ACP (Fig. 11) como el FDC (Fig. 12) discriminaron cinco grupos, correspondientes a L. tucumana, R. cuprinus, R. tenellus, Z. zulmae y Z. gordurasensis. Otro grupo quedó conformado por R. promatensis y R. amancaezensis. En el ACP, el componente principal I $(\mathrm{CPI})$ respondió por el $86,52 \%$ de la variación, siendo la mayor responsable la variación en el diámetro mayor de la conchilla; el componente principal II (CPII) respondió por el 6,84\%, siendo los responsables la variación en el diámetro del ombligo, seguido por la contribución negativa del número de anfractos (Tabs VIII y IX). En el análisis multivariado independiente del tamaño (FDC), los dos primeros factores contribuyeron por el $88,6 \%$, interviniendo principalmentela variación en el número de anfractos y en el diámetro del ombligo (Tabs X y XI).

Tabla VIII. Variación total explicada en el Análisis de Componentes Principales.

\begin{tabular}{cccc}
\hline Componente & Total & \% variación & \% Acumulado \\
\hline 1 & 5,19 & 86,52 & 86,52 \\
2 & 0,41 & 6,84 & 93,36 \\
3 & 0,18 & 3,05 & 96,41 \\
4 & 0,13 & 2,24 & 98,65 \\
5 & 0,05 & 0,90 & 99,56 \\
6 & 0,03 & 0,44 & 100,00 \\
\hline
\end{tabular}

Tabla IX. Matriz de coeficiente de "escores" de los cuatro primeros Componentes Principales.

\begin{tabular}{lcccc}
\hline \multirow{2}{*}{ Variable } & \multicolumn{4}{c}{ Componente } \\
\cline { 2 - 5 } & 1 & 2 & 3 & 4 \\
\hline Altura de la abertura & 0,1809 & 0,3458 & $-0,5676$ & $-2,0919$ \\
Altura & 0,1877 & $-0,2829$ & $-0,4975$ & $-0,1043$ \\
Diámetro de la abertura & 0,1803 & $-0,4130$ & $-1,1602$ & 1,4499 \\
Diámetro mayor & 0,1889 & 0,2348 & $-0,1632$ & 0,1750 \\
Diámetro del ombligo & 0,1675 & 1,0656 & 1,0199 & 0,9160 \\
Número de anfractos & 0,1686 & $-0,9361$ & 1,5727 & $-0,2942$ \\
\hline
\end{tabular}

Tabla X. Variación total explicada en el análisis de Funciones Discriminantes Canónicas.

\begin{tabular}{cccc}
\hline Función & Eigenvalue & \% variación & \% acumulado \\
\hline 1 & 18,430 & 55,46 & 55,46 \\
2 & 11,020 & 33,16 & 88,62 \\
3 & 2,360 & 7,11 & 95,74 \\
4 & 0,740 & 2,20 & 97,94 \\
5 & 0,650 & 1,95 & 99,89 \\
6 & 0,035 & 0,11 & 100,00 \\
\hline
\end{tabular}

Tabla XI. Coeficientes estandarizados de las cuatro primeras Funciones Discriminantes Canónicas.

\begin{tabular}{lcccc}
\hline \multirow{2}{*}{ Variable } & \multicolumn{4}{c}{ Componente } \\
\cline { 2 - 5 } & 1 & 2 & 3 & 4 \\
\hline Altura de la abertura & 0,4118 & $-0,5473$ & 0,9305 & $-0,5421$ \\
Altura & 0,5074 & 0,8487 & 0,8272 & 0,5632 \\
Diámetro de la abertura & $-0,1461$ & 0,3211 & $-1,1524$ & 0,2825 \\
Diámetro mayor & 0,1547 & 0,1656 & $-1,1777$ & $-1,2606$ \\
Diámetro del ombligo & 0,9695 & $-1,1437$ & 0,1641 & 0,8673 \\
Número de anfractos & $-1,0115$ & 0,7749 & 0,5034 & 0,2696 \\
\hline
\end{tabular}

Zilchogyra zulmae, que es la especie de mayor tamaño de las aquí estudiadas (Fig. 13), quedó discriminada de $Z$. gordurasensis en el análisis multivariado, tanto por el número de vueltas como por el diámetro del ombligo (Figs 11 y 12). Las especies de Radiodiscus, que son las más pequeñas junto con $R$. amancaezensis y $Z$. gordurasensis, quedaron mejor discriminadas por la variación en el diámetro del ombligo (Figs 11, 12 y 14), siendo que R. promatensis sp. nov. tiene, proporcionalmente, el ombligo de mayor diámetro, seguido por R. tenellus y R. cuprinus. La conchilla deL. tucumana es la más diferente de las especies aquí estudiadas, con ombligo amplio y mayor número de anfractos (Fig. 14).

\section{Comentarios biogeográficos}

Como se ha indicado, la región paranense o Provincia Subtropical Oriental, donde está ubicado el "Centro de Pesquisas e Conservação da Natureza Pró-Mata", comprende el extremo sur de Brasil (al oeste de la Serra do Mar hasta el centro de Rio Grande do Sul), el extremo nordeste de Argentina y el este de Paraguay.

En losúltimos años varias especies de Punctoidea fueron halladas en el extremo sur de Brasil, Zilchogyra discoidea, Radiodiscus costellifer, Radiodiscus (Radiodiscus) thomei, Radiodiscus (Radiodiscus) compactus, Radiodiscus (Radiodiscus) bolachaensis y Radioconus (Radioconus) amoenus (Thiele, 1927) (Á.L.M. FonseCA, com. pers.). En Pro-Mata uno de los autores (JWT) encontró Ptychodon schuppi (Suter, 1900) (? = Rotadiscus

Revista Brasileira de Zoologia 21(4): 925-935, dezembro 2004 

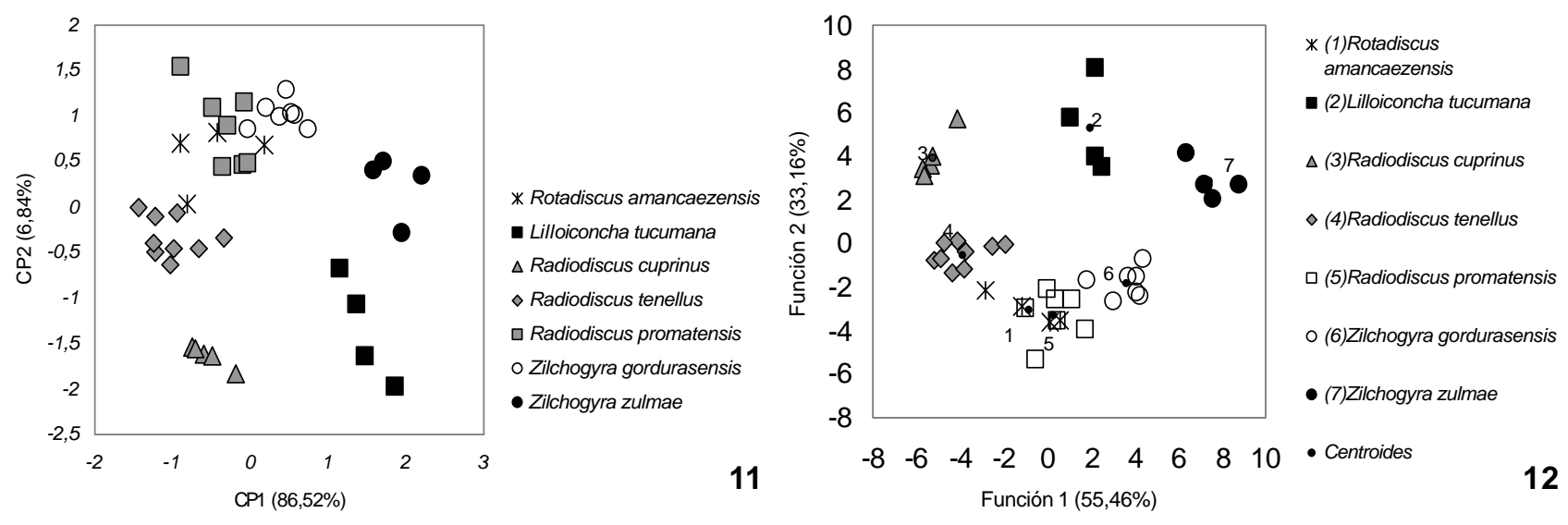

Figuras 11-12. (11) Gráfico de dispersión de los dos primeros componentes principales para siete especies de Charopidae del CPCN/ PRO-MATA; (12) Gráfico de dispersión de las dos primeras funciones discriminantes canónicas para siete especies de Charopidae del CPCN/PRO-MATA.
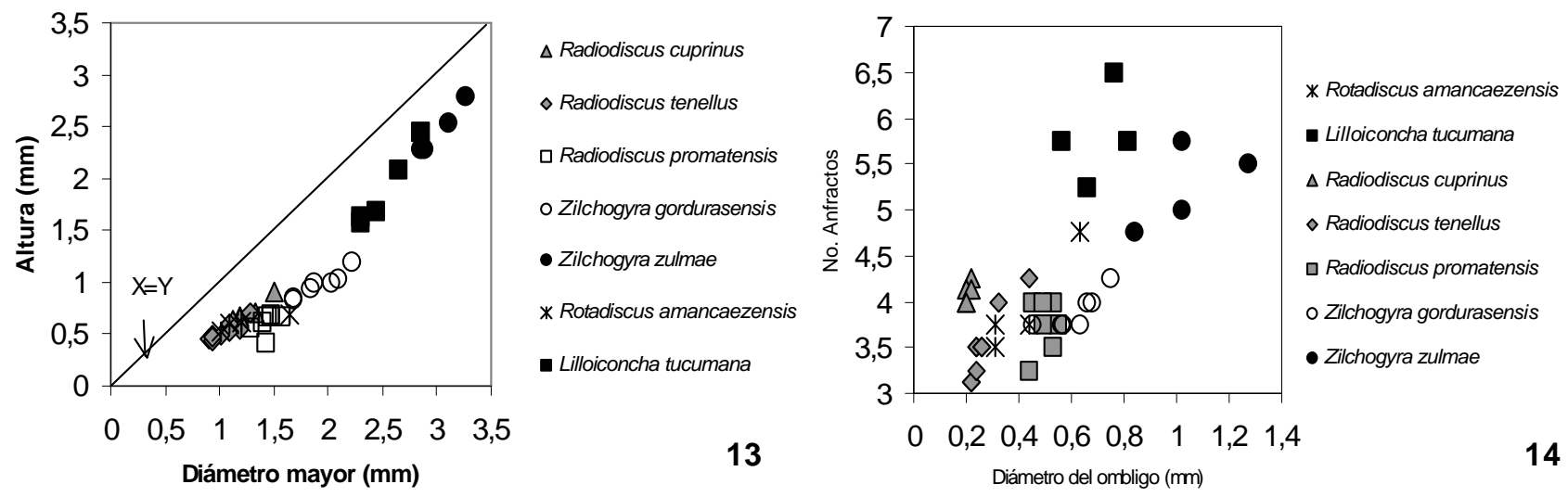

Figuras 13-14. (13) Variación de la altura en relación al diámetro mayor de la conchilla de siete especies de Charopidae del CPCN/PROMATA; (14) Gráfico de dispersión de diámetro del ombligo por número de anfractos de la conchilla de siete especies de Charopidae del CPCN/PRO-MATA.

amancaezensis), Radiodiscus amoenus, Radiodiscus cuprinus y Retidiscus reticulatus Fonseca \& Thomé, 1995 y Trochogyra gordurasensis. Todas ellas fueron descriptas originalmente para el sur de Brasil o el este de Paraguay, aunque algunas de sus identidades y combinaciones deben ser confirmadas.

Según Cabrera \& Wiluink (1973) la selva de las yungas abarca las laderas oriental es de la Cordillera de los Andes, formando una faja angosta desde Venezuela hasta el noroeste argentino. En la Argentina, la selva de yungas se desarrolla de manera discontínua por las quebradas y montañas del extremo norte de Salta, este de Jujuy y, de nuevo, por el centro de Salta, siguiendo por el centro de Tucumán hasta el este de Catamarca. Su clima es cálido y húmedo, con Iluvias abundantes, principalmente durante el verano, y heladas en el invierno. Las precipitaciones anuales alcanzan los $2.000 \mathrm{~mm}$, en tanto que la temperatura media varía de 14 a $21^{\circ} \mathrm{C}$. El suelo es ácido, humífero, generalmente con roca a medio desintegrar y capas de detritus vegetales más o menos espesa. El tipo de vegetación predominante es la selva montana, pero también hay bosques montanos, praderas, etc., estando muy relacionada a la Provincia Subtropical Oriental (CABRERA 1958).

Meyer \& Weyrauch (1966) citan para la selva húmeda subtropical de la provincia de Tucumán (ubicada entre los 600 y 1500 msnm) a L. tucumana, R. crenulatus, R. amancaezensis y Zilchogyra gordurasensis vivientes en capas de hojarasca y tierra vegetal de un espesor de 20 a $50 \mathrm{~cm}$. Se desarrollan, también, Scutalus tupacii (d'Orbigny, 1835), un Orthalicidae de mediano tamaño, y especies de los géneros Systrophia Pfeiffer, 1855 (Systrophiidae) y Habroconus Fischer \& Crosse, 1872 (Euconulidae). 
De acuerdo a Cabrera \& Willink (1973) la fauna de invertebrados de las provincias occidental y oriental contiene muchas especies en común. En esta oportunidad, dicha opinión puede ser verificada para L. tucumana, R. amancaezensis y $Z$. gordurasensis. $R$. tenellus extiende su distribución desde el este de Paraguay, en tanto queR. cuprinus, R. promatensis y Z. zulmae al presente sólo se han registrado en Pro-Mata.

Según MeYer \& WeYRAuch (1966), aparece en la provincia deTucumán, además, una especie de gasterópodo introducido, muy frecuentea lo largo de las rutas: Radiodiscus pilsbryi Hylton Scott, 1957. De acuerdo a HAUSDORF (2002), esta especie es la misma que Paralaoma servilis (Shuttleworth, 1852), un Punctidae de amplia distribución antrópica en el mundo. En América del Sur ha sido encontrada por dicho autor en Colombia; en Perú, por Flórez Bustamante (1968) y en Argentina, por WeYrauch (1965b). En nuestro continente ha sido descripta varias veces como especie nueva (R. pilsbryi, Zilchogyra cleliae Weyrauch, 1965 y Radiodiscus misionensis Hylton Scott, 1963) y citada con diferentes combinaciones (HYLTON ScotT 1973, Fernández \& M orRis 1986, Fonseca \& Thomé 1995b, Miquel $\&$ PARENT 1997).

\section{AGRA DECIMIENTOS}

A los becarios y practicantes del Laboratorio de Malacologia de la PUCRS que colaboraron con la colecta y separación del material biológico, en especial a J. Picanço, P. Bergonci, S. Gomes y L. da Silva. A.E. Moyses por la elaboración de la figura 1. S.E. Miquel pertenece a la Carrera del Investigador Científico del Consejo Nacional de Investigaciones Científicas y Técnicas. J.W. Thomé recibió becas y auxilios del CNPq y FAPERGS. El Proyecto fue parcialmente financiado por FAPERGS y por un Convenio de Cooperación Binacional Argentino-brasileño (Conicet-CNPq) (Res. Conicet No. 1129/97; CNPq Proc. No. 910154/96-1).

\section{REFERENCIAS BIBLIOGRÁ FICAS}

BAKER, H.B. 1928. Thiele's Brazilian land snails. The Nautilus, Philadel phia, 41 (4): 124-129.

Bertoletti, J.J. \& M.B. Teixeira. 1995. Centro de Pesquisas e Conservação da Natureza Pró-Mata. Termo de referencia. Divulgações do Museu de Ciências e Tecnologia - UBEA/ PUCRS, Porto Alegre, 2: 1-47.

Cabrera, A.L. 1958. Fitogeografía, p. 103-207. In: F. de Aparicio \& H.A. DifRIERI (Dirs). La Argentina. Suma de Geografía. Buenos Aires, Peuser Ed., 369p.

Cabrera, A.L. \& A. Willink. 1973. Biogeografía de América Latina. Washington, D.C., Secretaría General de la Organización delos EstadosAmericanos, SerieBiología, Monografía 13, 120p.

Climo, F.M. 1989. The panbiogeography of New Zealand as illuminated by the genus Fectola I redale, 1915 and subfamily Rotadiscinae Pilsbry, 1927 (Mollusca: Pulmonata: Punctoi- dea: Charopidae). New Zeal and Journal of Zoology, Wellington, 16: 587-649.

EMBERTON, K.C. 1985. Seasonal changes in the reproductive gross anatomy of the land snails Triodopsis tridentata tridentata (Pulmonata: Polygyridae). Malacologia, Ann Arbor, 26 (12): 225-239.

FERnÁndeZ, D. 1973. Catálogo de la malacofauna terrestre argentina. La Plata, Comisión de Investigaciones Científicas, Monografías 4, 197p.

Fernández, D. \& M.R. Morris. 1986. Presencia de Zilchogyra cleliae Weyrauch, 1965 (Mollusca-Pulmonata) para la provincia de Santa Fe (República Argentina) y aportes al conocimiento de su ultraestructura conquiliar. Notas del Museo de La Plata, Zoología, La Plata, 21 (204): 23-29.

Flórez Bustamante, A.E. 1968. Contribución al conocimiento de los gasterópodos de la región del Cusco. Revista de la Facultad de Ciencias, Cuzco, 2: 69-127.

FonseCA, Á.L.M. dA \& J.W. Thomé. 1993a. Descrição de Glabrogyra subgen. n., recaracterização de Austrodiscus twomeyi (Parodiz, 1954) e reclassificação das espécies sulamericanas dos géneros Austrodiscus Parodiz 1957, Radioconus Baker, 1927, Radiodomus Baker, 1930 e Trochogyra Weyrauch 1965 (Charopidae) e Zilchogyra Weyrauch 1965 (Helicodiscidae) (Gastropoda, Stylommatophora, Endodontoidea). Iheringia, Série Zoologia., Porto Alegre, 75: 97-105.

. 1993b. Classificação das espécies neotropicais de micromoluscos dos géneros Stephanoda Albers 1860, Stephadiscus Scott 1981 e Ptychodon Ancey 1888 (Charopidae, Endodontoidea, Stylommatophora, Gastropoda). Acta Biologica Leopoldensia, São Leopoldo, 15 (2): 65-76.

. 1994. Descrição de Radiodiscus vazi, sp. n. de São Paulo, Brasil, com proposição de recombinação de duas outras espécies sob este gênero e R. bolachaensis, nom. n. e sinonimização de uma espécie de Radioconus (Gastropoda, Stylommatophora, Charopidae). Revista Brasileira de Zoologia, Curitiba, 11 (2): 265-275.

. 1995a. A new genus and species of Charopidae (Gastropoda, Endodontoidea, Stylommatophora), Retidiscus reticulatus, from Southern Brazil. Walkerana, Ann Arbor, 7 (17/18): 55-62. [1993-1994]

. 1995b. Recaracterização do subgênero Toltecia Pilsbry com descriçào da conquiliomorfologia e anatomia dos sistemas excretor e reproductor de Punctum (Toltecia) pilsbryi (Scott), comb. n. (Gastropoda, Stylommatophora, Punctidae). Revista Brasilera de Zoologia, Curitiba, 12 (1): 189-209.

. 2000. Conquiliomorfología y anatomía de los sistemas excretor y reproductor de Radiodiscus cuprinus sp. $\mathrm{n}$. (Gastropoda: Stylommatophora: Charopidae). Neotropica, La Plata, 46: 11-18.

HAUSDORF, B. 2002. Introduced Iand snails and slugs in Colombia. Journal of Molluscan Studies, London, 68: 127131. 
HIDALGO, J.G. 1869. Description d'espéces nouvelles. Journal de Conchyliologie, Paris, (3) 9 (17): 410-413.

. 1870. Catalogue des Coquilles terrestres recueillies par les natural istes de la commission scientifique espagnole sur divers points de I'Amérique méridionale. Journal de Conchyliologie, Paris, (3) 9 (18): 27-70.

Hylton ScotT, M.I., 1948. Moluscos del noroesteargentino. Acta Zoológica Lilloana, San Miguel de Tucumán, 6: 241-274. . 1957. Endodóntidos neotropicales II (Moll. Pulm.). Neotropica, La Plata, 3 (12): 79-87. 1963. Tres nuevos Endodóntidos de Tucumán. Neotropica, La Plata, 9 (29): 49-54.

— . 1973. Endodóntidos neotropicales V (Gastropoda Pulmonata). Neotropica, La Plata, 19 (60): 126-131.

Meyer, T. \& W.K. Weyrauch. 1966. Guía para dos excursiones biológicas en la provincia de Tucumán. San Miguel de Tucumán, Universidad Nacional de Tucumán, Miscelánea 23, 126p.

Miquel, S.E. \& H. Parent. 1997. Moluscos Gasterópodos de la provincia de Santa Fe (Argentina). Malacological Review,
Michigan, 29: 107-112.

PILSBRY, H.A. \& J.H. FerRIS. 1906. Mollusca of the Southwestern States. II. Proceeding of Academy of natural Sciences of Philadel phia, Philadel phia, 58: 123-175.

QUINTANA, M.G. 1982. Catálogo preliminar de la malacofauna del Paraguay. Revista del Museo argentino de Ciencias natural es "Bernardino Rivadavia", Buenos Aires, Zoología, 11 (3): 61-158.

Solem, A. 1976. Endodontoid land snails from Pacific Islands (Mollusca: Pulmonata: Sigmurethra). Chicago, Field Museum of Natural History, vol. 1, XII+508p.

THIELE, J. 1927. Über einige brasilianische Landschnecken. Abhandlungen der Sencken bergischen Naturforschenden Gesellschaft, Frankfurt am Main, 40 (3): 307-399.

Weyrauch, W.K. 1965a. Neue und verkannte Endodontiden aus Südamerika. Archiv für Molluskenkunde, Frankfurt am Main, 94 (3/4): 121-134.

. 1965b. Cinco nuevos Endodóntidos de Argentina y Perú (Gastropoda, Euthyneura). Neotropica, La Plata, 11 (36): 105-115.

Recebido em 20.I.2004; aceito em 12.XI.2004. 